\title{
Updated assessment of cystic fibrosis mutation frequencies in non-Hispanic Caucasians
}

\author{
Glenn E. Palomaki, BS ${ }^{1}$, James E. Haddow, $\mathrm{MD}^{1}$, Linda A. Bradley, $P h D^{2}$, and Stacey C. FitzSimmons, PhD ${ }^{3}$
}

\begin{abstract}
Purpose: To update estimates of individual and cumulative cystic fibrosis (CF) mutation frequencies in nonHispanic Caucasians for the prenatal screening panel recommended by American College of Medical Genetics and to determine the impact on screening performance. Methods: Two data sources were used. In the first (CF Genetic Analysis Consortium), our re-analysis was restricted to North American studies. In the second (CF Foundation National Patient Registry), we performed a new analysis restricted to individuals tested at eight Therapeutic Development Network sites. Results: The updated average cumulative proportion of mutations identified is $88.34 \%$ (higher than previously reported), indicating that $78 \%$ of high-risk couples (and affected fetuses) can potentially be identified. Conclusion: Prenatal CF screening in U.S. non-Hispanic Caucasians is more effective than previously thought. Genet Med 2002:4(2):90-94.
\end{abstract}

Key Words: cystic fibrosis, mutation frequencies, Caucasians, prenatal screening

In 1997, a Consensus Development Conference convened by the National Institutes of Health (NIH) recommended that prenatal screening for cystic fibrosis be implemented in the United States. ${ }^{1}$ As a follow-up, implementation issues were addressed in 1998 by a second NIH sponsored conference ${ }^{2}$ and at a working conference in Scarborough, Maine. ${ }^{3}$ Recently, the American College of Medical Genetics (ACMG) published laboratory standards and guidelines for prenatal cystic fibrosis screening, including a recommended core testing panel of 25 mutations. ${ }^{4,5}$ When estimating the proportion of carrier couples (or affected fetuses) who can be identified by such a panel, it is first necessary to derive mutation frequencies from a large, unbiased sampling of individuals with clinically defined cystic fibrosis whose race/ethnicity are known. The ACMG laboratory guidelines provide an estimated cumulative frequency of $80 \%$ for Caucasians of European heritage but do not include individual estimates of mutation frequencies. In this study, we re-analyze two large existing data sources ${ }^{6,7}$ to verify and extend this estimate of the panel's performance. ${ }^{4}$ Our analysis focuses on mutation frequencies in non-Hispanic Caucasians. This group is at high risk for cystic fibrosis. The prevalence of cystic fibrosis, and also the distribution of mutations causing disease, vary by racial/ethnic background. Nearly all of the data used in the current study are based on individuals with cystic fibrosis who reside in the United States. The purpose of this analysis is to provide additional insight on the distribution of

From the ${ }^{1}$ Foundation for Blood Research, Scarborough, Maine; ${ }^{2}$ Clinigene Laboratories, Hauppauge, New York; and ${ }^{3}$ FitzSimmons and Associates, Chevy Chase, Maryland.

Glenn E. Palomaki, Foundation for Blood Research, 69 U.S. Route One, PO Box 190, Scar-

borough, ME 04074-0190.

Received: September 19, 2001.

Accepted: November 27, 2001. cystic fibrosis mutations to help better define the clinical validity of prenatal screening.

\section{MATERIALS AND METHODS}

Published reports of mutation frequencies in individuals with cystic fibrosis were selected, based on the following criteria: (1) all (or most) of the mutations in the recommended panel were analyzed, (2) a large number of individuals was studied, (3) some measure of race/ethnicity was available, and (4) a high proportion of the individuals was from the United States. Four possible data sources were examined for suitability. ${ }^{6-9}$ One of these reported frequencies only for European or African countries. ${ }^{8}$ Another could have been used, ${ }^{9}$ but a proportion of its data was already included in an earlier, collaborative study $y^{6}$ and appeared to be biased toward underestimating the frequency of delF508. The two remaining data sources were suitable for use in analysis. ${ }^{6,7}$

Data from the CF Consortium were obtained directly from that publication's Table 1 but were restricted to studies from North America. ${ }^{6}$ Additional information gathered from laboratories since publication is available on-line at $w w w$. genet.sickkids.on.ca/cftr/freqtables.html. However, it is not clear whether blank entries on these newer tables indicate "tested for and none found" or "not tested" (personal communication, D. Markiewicz). For this reason, the on-line information is not included.

The CF Foundation has supported a registry since 1966, and collection methods have been reported elsewhere. ${ }^{10} \mathrm{~A}$ total of 20,077 patients was seen at 114 CF Foundation-accredited CF care centers in the United States in 1999. Each center is required to report annually to the CF Foundation Patient Registry the results of a standardized questionnaire that includes mutation testing results and race/ethnicity for each patient 
Table 1

Cystic fibrosis (CF) mutation frequencies for non-Hispanic Caucasians in the United States within the recommended 25 mutation panel

\begin{tabular}{|c|c|c|c|c|c|}
\hline \multirow[b]{2}{*}{ No. } & \multirow[b]{2}{*}{ Mutation } & \multicolumn{4}{|c|}{ Mutation frequency (\%) } \\
\hline & & CF Consortium $^{a}$ & CF Foundation ${ }^{b}$ & Average & Cumulative \\
\hline 1 & delF508 & 68.94 & 75.90 & 72.42 & 72.42 \\
\hline 2 & G542X & 2.17 & 2.39 & 2.28 & 74.70 \\
\hline 3 & G551D & 2.06 & 2.44 & 2.25 & 76.95 \\
\hline 4 & $621+1 G>T$ & 1.92 & 1.22 & 1.57 & 78.52 \\
\hline 5 & W1282X & 1.42 & 1.57 & 1.50 & 80.02 \\
\hline 6 & N1303K & 1.32 & 1.22 & 1.27 & 81.29 \\
\hline 7 & R553X & 0.97 & 0.76 & 0.88 & 82.15 \\
\hline 8 & delI507 & 0.25 & 1.50 & 0.87 & 83.03 \\
\hline 9 & $\mathrm{R} 117 \mathrm{H}$ & 0.84 & 0.56 & 0.70 & 83.73 \\
\hline 10 & $3849+10 \mathrm{kbC}>\mathrm{T}$ & 0.73 & 0.43 & 0.58 & 84.31 \\
\hline 11 & $2789+5 G>A$ & 0.48 & 0.48 & 0.48 & 84.79 \\
\hline 12 & $1717-1 \mathrm{G}>\mathrm{A}$ & 0.54 & 0.41 & 0.48 & 85.26 \\
\hline 13 & $\mathrm{R} 347 \mathrm{P}$ & 0.46 & 0.43 & 0.45 & 85.71 \\
\hline 14 & $711+1 G>T$ & 0.77 & 0.08 & 0.43 & 86.13 \\
\hline 15 & $\mathrm{R} 560 \mathrm{~T}$ & 0.30 & 0.46 & 0.38 & 86.51 \\
\hline 16 & A455E & 0.54 & 0.13 & 0.34 & 86.85 \\
\hline 17 & 3659delC & 0.32 & 0.36 & 0.34 & 87.19 \\
\hline 18 & G85E & 0.38 & 0.20 & 0.29 & 87.48 \\
\hline 19 & R1162X & 0.09 & 0.36 & 0.23 & 87.70 \\
\hline 20 & 2184delA & 0.10 & 0.23 & 0.17 & 87.87 \\
\hline 21 & $1898+1 \mathrm{G}>\mathrm{A}$ & 0.09 & 0.23 & 0.16 & 88.03 \\
\hline 22 & $\mathrm{R} 334 \mathrm{~W}$ & 0.13 & 0.15 & 0.14 & 88.17 \\
\hline 23 & $\mathrm{I} 148 \mathrm{~T}$ & 0.10 & 0.08 & 0.09 & 88.26 \\
\hline 24 & $3120+1 \mathrm{G}>\mathrm{A}$ & 0.10 & 0.05 & 0.08 & 88.33 \\
\hline 25 & 1078delT & 0.03 & 0.00 & 0.02 & 88.35 \\
\hline Cumulative frequency & & 85.05 & 91.64 & 88.35 & \\
\hline
\end{tabular}

Boldface type indicates that the mutation was observed more than 10 times. Italic type indicates an arbitrary assignment of $0.10 \%$ because those mutations were not included in the report.

${ }^{a}$ Cystic Fibrosis Genetic Analysis Consortium, ${ }^{6}$ based on between 2,187 and 9,792 cystic fibrosis chromosomes.

${ }^{b}$ Based on a new analysis of the Cystic Fibrosis Foundation National Patient Registry based on 3,938 chromosomes.

seen. The questionnaire lists 23 of the 25 core panel mutations individually. Frequencies of the other two mutations have been derived from a detailed process of review and recoding directed by one of the authors (SCF). Data for the present analysis were limited to individuals with cystic fibrosis who were seen at eight Therapeutic Development Network (TDN) sites that specialize in clinical research. These TDN sites receive funding from the CF Foundation that provides genotyping at no cost for patients whose mutation information is incomplete. Beginning in 1998, a concerted effort has been made to genotype all patients. Laboratories providing this service offer extensive panels that include the 25 mutations in the recommended panel.

\section{RESULTS}

Table 1 shows the frequencies of the 25 mutations recommended by ACMG for preconception and prenatal screening, based on a reanalysis of the CF Consortium data ${ }^{6}$ and a new analysis, using patient data collected by the CF Foundation. ${ }^{7}$ The table is restricted to non-Hispanic Caucasians with clinically defined cystic fibrosis, and the mutations are listed in order of decreasing average frequency. As a measure of reliability, bolded entries indicate that the mutation was observed more than 10 times and, for the CF Consortium frequencies, the mutation was tested by more than one quarter of the participating members. The fifth column lists the arithmetic aver- 
age of individual estimates from the two sources. The average mutation frequency is used to compute the cumulative proportion (column 6) both to be conservative and to increase the accuracy and precision of the estimates for the least common mutations.

The most striking difference between the two mutation frequency estimates in Table 1 is for the most common mutation, delF508. The estimate from the CF Consortium (68.94\%) is nearly 7 percentage points lower than the CF Foundation's estimate $(75.90 \%)$. This difference is statistically significant $\left(\chi^{2}\right.$ $>200, P<0.001)$. The difference in the cumulative frequency is mainly due to this difference; the cumulative frequency for the 25 mutations is 6.59 percentage points higher, when derived from the CF Foundation National Patient Registry.

The CF Consortium 6 reported the frequency for 22 of the 25 recommended mutations. Frequencies for the three mutations not reported (2184delA, I148T, and $3120+1 \mathrm{G}>\mathrm{A}$ ) are relatively uncommon and have been arbitrarily listed as $0.10 \%$ (italicized entries, Table 1). The cumulative frequency of $85.05 \%$ for the re-analyzed CF Consortium data are higher than originally reported for North America (79.9\%), ${ }^{6}$ for two reasons. First, the original report summarized all data from North America, without taking race/ethnicity into account. We have removed the last 13 studies reported in the original article listed under "North America." Those studies focused on Hispanic, Ashkenazi Jewish, or African American individuals. Second, the original report divided the observed number of times that each mutation was identified by the total number of chromosomes reported for all studies rather than the number of chromosomes actually tested for that mutation. This oversight was mentioned in the CF Consortium report and has since been corrected. ${ }^{11}$ The present analysis uses mutationspecific denominators, restricted to the number of chromosomes that were tested for the mutation in question. For the more common mutations, this second correction has little or no impact. However, for the less common mutations, the corrected frequencies are, in some instances, considerably higher. For example, the mutation frequency for A $455 \mathrm{E}$ is $0.28 \%$ before correction and $0.54 \%$ after.
The CF Foundation National Patient Registry contains information from 20,077 cystic fibrosis patients in the United States who visited one of 114 CF care centers in 1999. Until now, mutation frequency analyses derived from that registry have included the entire collection of genotypes. Such an approach has strength in numbers. Unfortunately, it is not possible to document which and how many mutations have been included in testing panels used by the contributing centers. Thus a small observed number of an uncommon mutation might either indicate low frequency or that few patients had been tested for that mutation. To avoid potential problems in interpreting results, the present analysis is restricted to the 2,605 patients attending one of the eight TDN sites (see Materials and Methods). These sites account for approximately $16 \%$ of all non-Hispanic Caucasians in the registry. We compared the mutation frequencies in the TDN group with all non-Hispanic Caucasians in the registry, to determine whether any differences exist. Overall, the mutation frequencies and the cumulative proportion of mutations identified are similar, including the frequency of delF508 (75.90\% in the TDN group and $75.04 \%$ in the registry as a whole).

Existing reports of mutation frequencies that use the $\mathrm{CF}$ Foundation National Patient Registry do not stratify Caucasian results into Hispanic and non-Hispanic categories. ${ }^{12}$ The registry does, however, include some information about race/ ethnicity that allows this analysis to be done. Of the 2,507 individuals with cystic fibrosis who declared that they were Caucasian, 2,130 (85\%) were non-Hispanic, 75 (3\%) were Hispanic, and the remaining 302 (12\%) were of unknown heritage. Genotypes are available for 1,969 (92.4\%) of the nonHispanic Caucasians. The CF Foundation's registry does not allow separate identification of patients who are Ashkenazi Jewish.

Table 2 shows the cumulative proportion of mutations identified and the carrier couple detection rate (the square of the cumulative proportion of mutations identified) for 1, 5, 10, 15, 20, and 25 mutations. Average mutation frequencies are added in the order shown in Table 1. The third and fifth columns show marginal gains in the proportion of mutations identified

Table 2

Comparison of cystic fibrosis mutation panel size and proportion of carrier couples detected, assuming an analytic sensitivity of $100 \%$

\begin{tabular}{|c|c|c|c|c|}
\hline $\begin{array}{l}\text { No. of } \\
\text { mutations } \\
\text { in the } \\
\text { panel }\end{array}$ & $\begin{array}{l}\text { Cumulative } \\
\text { proportion of } \\
\text { mutations } \\
\text { identified }^{a}(\%)\end{array}$ & $\begin{array}{c}\text { Marginal gain } \\
\text { in proportion } \\
\text { of mutations } \\
\text { identified }^{b}\end{array}$ & $\begin{array}{l}\text { Cumulative } \\
\text { proportion of } \\
\text { carrier couples } \\
\text { detected }(\%)\end{array}$ & $\begin{array}{l}\text { Marginal gain } \\
\text { in carrier } \\
\text { couple } \\
\text { detection }^{b}\end{array}$ \\
\hline 1 & 72.42 & & 52.4 & \\
\hline 5 & 80.02 & +7.6 & 64.0 & +11.6 \\
\hline 10 & 84.31 & +4.3 & 71.1 & +7.1 \\
\hline 15 & 86.51 & +2.2 & 74.8 & +3.7 \\
\hline 20 & 87.87 & +1.3 & 77.1 & +2.3 \\
\hline 25 & 88.35 & +0.5 & 78.0 & +0.9 \\
\hline
\end{tabular}

${ }^{a}$ Mutations added in the order shown in Table 1.

${ }^{b}$ Difference in the cumulative proportion due to the mutations just added. 
and proportion of carrier couples detected that result from each successive addition of mutations. The term marginal gain is used to describe the difference in the cumulative proportions, due to the mutations just added. For example, the carrier couple detection rate for one mutation (delF508) is 52.4\%. With the addition of four more mutations, the detection rate increases to $64.0 \%$, a marginal gain of 11.6 percentage points. As more mutations are added, the marginal gain decreases. At all levels in Table 1, however, the increase in carrier couple detection is greater than the corresponding increase in the proportion of mutations identified. Adding 5 more mutations to an existing panel of 15 increases the proportion of mutations identified by approximately $1.32 \%$, but the corresponding increase in carrier couple detection is $2.3 \%$. These data demonstrate that the primary focus for judging feasibility of adding mutations ought to be the gain in carrier couples detected, rather than just the increase in the proportion of mutations identified.

\section{DISCUSSION}

Several hundred DNA tests are now available, or potentially available, for clinical testing in the United States. Varying levels of concern have been expressed about the uncritical introduction of these tests into medical practice. The Office of Genetics and Disease Prevention at the Centers for Disease Control and Prevention is supporting the development of a model system for the comprehensive collection, evaluation, and dissemination of information about genetic testing, as a step in evidencebased policy development. The four main components of this model system are analytic validity, clinical validity, clinical utility, and ethical legal and social implications. To evaluate the effectiveness of this model system, prenatal screening for cystic fibrosis has been chosen as the initial challenge, due to its imminent widespread introduction. This updated analysis of cystic fibrosis mutation frequencies was done as part of that evaluation, under the heading of clinical validity.

The mutation frequencies in the present report were derived from genotypes of individuals with clinically defined disease. This is an important feature to be aware of, because mutation frequencies in the general population are less well documented, especially for the uncommon mutations included in the ACMG recommended panel. The prevalence of the disorder can be used to estimate carrier frequency. Given the mutation frequencies in individuals with the disorder and the assumption that all of those mutations will cause significant disease, the expected carrier rates for each of the mutations can be derived. For example, if the prevalence of cystic fibrosis is $1: 2,500$ and $75 \%$ of the mutations in affected individuals are delF508, then a carrier with delF508 should be identified once in every 33 individuals tested in the general population. For the more common mutations, pilot trials have already confirmed these expectations, indicating that the assumption of high penetrance is correct. However, few published pilot trials have used expanded mutation panels such as the one being reviewed in this project. Evidence has emerged that at least one mutation
(R117H) in the expanded panel occurs considerably more often in the general population than predicted, indicating that it is not associated with the clinical features of cystic fibrosis in most instances. As a partial solution, reflexive testing has been recommended to identify those in whom the mutation could cause cystic fibrosis in the offspring in combination with a second disease-causing mutation. ${ }^{4,5}$ A second mutation (I148T) in the expanded panel is also being reported more frequently in the general population, again indicating low penetrance. ${ }^{13,14}$ These findings indicate that the composition of the panel may benefit from being revised. This demonstrates the importance of collecting information on the frequency of mutations in the women/couples undergoing prenatal screening for cystic fibrosis. This form of "epidemiological monitoring" provides a means to identify other mutations with low penetrance. Laboratories providing screening services ought to be encouraged to establish protocols to monitor performance.

One point that deserves further consideration is the marginal gain in detection afforded by increasing the cumulative proportion of mutations identified by a given panel. From the standpoint of prenatal screening, it is important to understand not only how often individuals with mutations (carriers) will be identified, but also, and even more important, how often carrier couples will be identified. Such couples are at a one in four risk of having an affected pregnancy. The marginal gains in detecting carrier couples are dependent on the extent of increase in the proportion of mutations identified (Table 2). These marginal gains also depend on the baseline proportion of mutations identified. For a stepwise 10 percentage point increase in the proportion of mutations identified over the baseline, the marginal gain in carrier couple detection can range from as little as $5 \%$ to as much as $17 \%$ (Table 3 ).

Mutation frequencies from the two data sources included in this study are in reasonable agreement, except for the frequency of delF508. One possible explanation for the low CF Consortium estimate is ascertainment bias. This bias could occur if some patients homozygous for delF508 were identified by testing in local laboratories, thereby avoiding the need for referral to laboratories providing more extensive, and expensive, testing. These referral laboratories are the type that participated in the CF Consortium. One laboratory that contributed substantially to the CF Consortium has reported that this type of ascertainment bias for delF508 exists in its data. ${ }^{9}$ This finding argues that the higher estimate derived from the CF Foundation National Patient Registry is more accurate.

The present analysis demonstrates that it can be useful to reexamine data from which a generally accepted conclusion has been derived and incorporated into policy. ${ }^{4}$ In this case, the revised estimate indicates that, at least in the non-Hispanic Caucasian population, the recommended policy will be even more effective than originally described. The results can be used by screening programs to help ensure that physicians and patients are receiving accurate and up-to-date information. Furthermore, the comparison of the two data sets in the present study has identified systematic differences that, when explored, has led to verifying an important source of bias. Al- 


\section{Table 3}

Marginal increases in the detection of cystic fibrosis (CF) carrier couples ${ }^{a}$ for a stepwise 10 percentage point increase in the cumulative proportion of mutations identified

\begin{tabular}{ccc}
\hline \multicolumn{2}{c}{$\begin{array}{c}\text { Proportion of CF mutations } \\
\text { identified (\%) }\end{array}$} & \\
\hline Initial (A) & Final $(\mathrm{A}+10)$ & $\begin{array}{c}\text { Marginal increase in CF } \\
\text { carrier couples detected }\end{array}$ (\%) \\
\hline 20 & 30 & 5 \\
30 & 40 & 7 \\
40 & 50 & 9 \\
50 & 60 & 11 \\
60 & 70 & 13 \\
70 & 80 & 15 \\
80 & 90 & 17 \\
\hline
\end{tabular}

${ }^{a}$ Carrier couples indicates that both partners are carriers of a cystic fibrosis mutation.

${ }^{b}$ Computed as $\left(\right.$ final $l^{2}-$ initial $\left.^{2}\right)$.

though the focus of this report is prenatal screening, the mutation frequency estimates are equally applicable to both newborn and diagnostic testing. Although time consuming, this type of comprehensive review can add useful information.

\section{Acknowledgments}

This study was largely funded by a cooperative agreement with the US Department of Health and Human Services, Centers for Disease Control and Prevention, Office of Genetics and Disease Prevention (UR3/CCU119356). We thank Preston Campbell, MD, from the Cystic Fibrosis Foundation for permission to use the CFF National Patient Registry, and Ase Sewall, a statistical programmer from Sewall, Inc., for her efforts performing essential data quality verifications for the race, ethnicity, and mutation variables. We also thank Ada
Hamosh, MD, for assistance with the interpretation and recoding of the less common mutations.

\section{References}

1. NIH Consensus Development Conference Statement. Genetic testing for cystic fibrosis. Available at: http://odp.od.nih.gov/consensus/cons/106/106 intro.htm. Accessed August 30, 2001.

2. Mennuti MT, Thomson E, Press N. Screening for cystic fibrosis carrier state. Obstet Gynecol 1999;93:456-461.

3. Haddow JE, Bradley LA, Palomaki GE, Doherty RA, Bernhardt BA, Brock DJ, Cheuvront B, Cunningham GC, Donnenfeld AE, Erickson JL, Erlich HA, Ferrie RM, FitzSimmons SC, Greene MF, Grody WW, Haddow PK, Harris H, Holmes LB, Howell RR, Katz M, Klinger KW, Kloza EM, LeFevre ML, Little S, Loeben G, McGovern M, Pyeritz RE, Rowley PT, Saiki RK, Short MP, Tabone J, Wald NJ, Wilker NL, Witt DR. Issues in implementing prenatal screening for cystic fibrosis: results of a working conference. Genet Med 1999;1:129-138.

4. Grody WW, Cutting GR, Klinger KW, Richards CS, Watson MS, Desnick RJ. Laboratory standards and guidelines for population-based cystic fibrosis carrier screening. Genet Med 2001;3:149-154.

5. Grody WW, Desnick RJ. Cystic fibrosis population carrier screening: Here at last are we ready? Genet Med 2001;3:87-90.

6. Kazazian HH for The Cystic Fibrosis Genetic Analysis Consortium. Population variation of common cystic fibrosis mutations. Hum Mutat 1994;4:167-177.

7. Cystic Fibrosis Foundation. Patient registry 1999. Annual data report. September 2000; Bethesda, Md;

8. Estivill X, Bancells C, Ramon C. Geographic distribution and regional origin of 272 cystic fibrosis mutations in European populations. Hum Mutat 1997;10:135-154.

9. Heim RA, Sugarman EA, Allitto BA. Improved detection of cystic fibrosis mutations in the heterogeneous U.S. population using an expanded, pan-ethnic mutation panel. Genet Med 2001;3:168-176.

10. FitzSimmons S. The changing epidemiology of cystic fibrosis. J Pediatr 1993;122: $1-9$.

11. Girogi S, Tandoi C, Ciminelli BM, Modiano G. A correction of the estimates of the least common cystic fibrosis (CF) mutations published by "The Cystic Fibrosis Genetic Analysis Consortium” in 1994. Gene Geogr 1997;11:57-59.

12. Hamosh A, FitzSimmons SC, Macek M, Knowles MR, Rosenstein BJ, Cutting GR. Comparison of the clinical manifestations of cystic fibrosis in black and white patients. J Pediatr 1998;132:25-29.

13. Wit DR, Coppinger J. Cystic fibrosis prenatal screening of 27,000 women in a large HMO. Am J Hum Genet 2001;69:A2.

14. Rohlfs EM, Sugarman EA, Heim RA, Allitto BA. Frequency of carriers of two cystic fibrosis mutations in an apparently unaffected adult population [abstract]. Genet Med 2001;3:237. 